\title{
Chinese Expert Consensus on Multidisciplinary Diagnosis and Treatment of Hepatocellular Carcinoma with Portal Vein Tumor Thrombus (2018 Edition)
}

\author{
Shuqun Cheng ${ }^{a} \quad$ Minshan Chen $^{b}$ Jianqiang Caic Juxian Sun ${ }^{a}$ Rongping Guo $^{b}$ \\ Xinyu $\mathrm{Bi}^{\mathrm{c}}$ Wan Yee Lau ${ }^{\mathrm{a}} \mathrm{d}$ Mengchao $\mathrm{Wu}^{\mathrm{a}}$ on behalf of Chinese Association of \\ Liver Cancer and Chinese Medical Doctor Association \\ a Department of Hepatic Surgery VI, Eastern Hepatobiliary Surgery Hospital, Second Military Medical \\ University, Shanghai, China; ${ }^{b}$ Department of Hepatobiliary Surgery, Sun Yat-sen University Cancer \\ Center, Guangzhou, China; ' Department of Hepatobiliary Surgery, Cancer Hospital, Chinese Academy

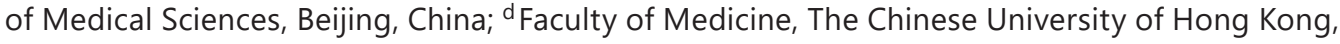 \\ Shatin, Hong Kong SAR, China
}

\section{Keywords}

Hepatocellular carcinoma - Portal vein tumor thrombus - Multidisciplinary therapy ·

Consensus

\begin{abstract}
Portal vein tumor thrombus (PVTT) is very common, and it plays a major role in the prognosis and clinical staging of hepatocellular carcinoma (HCC). We have published the first version of the Consensus in 2016. Over the past several years, many new evidences for the treatment of PVTT become available especially for the advent of new targeted drugs which have further improved the prognosis of PVTT. So, the Chinese Association of Liver Cancer revised the 2016 version of consensus to adapt to the development of PVTT treatment. Future treatment strategies for HCC with PVTT in China would depend on new evidences from more future clinical trials.

Prof. Cheng Shuqun

Department of Hepatic Surgery VI, Eastern Hepatobiliary Surgery Hospital

Second Military Medical University, Changhai Road 168

Yangpu District, Shanghai 200433 (China)

E-Mail chengshuqun@aliyun.com

Prof. Chen Minshan

Department of Hepatobiliary Surgery, Sun Yat-sen University

Cancer Center

651 Dongfeng Road East

Guangzhou 510060 (China)

E-Mail chminsh@mail.sysn.edu.cn
Prof. Cai Jianqiang

Department of Hepatobiliary Surgery, Cancer Hospital

Chinese Academy of Medical Sciences, Panjiayuan

Chaoyang District, in the South, 17th, Beijing 100021 (China)

E-Mail caijianqiang188@ sina.com 


\section{Liver Cancer}

\section{Introduction}

Hepatocellular carcinoma (HCC) is the sixth most prevalent cancer worldwide, and China accounts for more than half of new cases and deaths related to HCC every year [1]. The latest data indicated that the morbidity and mortality rates of HCC ranked the fourth and third, respectively, among all malignant tumors reported in China [2]. Given the advances in diagnosis and treatment strategies for different stages of HCC, the prognosis of HCC patients has improved. Unfortunately, $70-80 \%$ of patients are still diagnosed at an advanced stage as there are no obvious clinical symptoms at early stages. At present, the overall prognosis of HCC is not satisfactory.

Owing to the biological characteristics of liver cancer and the anatomical characteristics of the liver, HCC is prone to invade intrahepatic vessels, especially the portal venous system. In China, the incidences of portal vein tumor thrombus (PVTT) have been reported to range from 44 to $62.2 \%$ [3]. Once developed, PVTT progresses rapidly to cause portal hypertension, hepatocellular jaundice, and intractable ascites. The median survival of HCC patients with main PVTT is 2.7 months [4]. PVTT plays a major role in the prognosis and clinical staging of HCC $[5,6]$.

There have been no worldwide consensuses or guidelines on the diagnosis and treatment of HCC with PVTT. Guidelines in Europe and America follow the Barcelona Clinic Liver Cancer Staging (BCLC) and regard HCC with PVTT to be at BCLC Stage C. The guidelines also recommend treating HCC patients with PVTT with molecular-targeted drugs such as Sorafenib and Lenvatinib [7]. On the contrary, experts from Southeast Asian countries including China opine that multidisciplinary therapy including surgery, transcatheter arterial chemoembolization (TACE), radiotherapy (RT), and/or molecular-targeted drugs should be considered to achieve more satisfactory outcomes. But the difference is that Chinese doctors tend to use more curable treatments for the same subgroup of PVTT patients.

In May 2016, the Chinese National Research Cooperative Group for Diagnosis and Treatment of HCC with Tumor Thrombus launched The Chinese Expert Consensus on Multidisciplinary Diagnosis and Treatment of HCC with PVTT (version 2016) [8] based on the existing evidences published internationally and in China at that time. This version (version 2016) has been widely used and recognized clinically.

Over the past several years, many new evidences for the treatment of PVTT become available especially for the advent of new targeted drugs which have further improved the prognosis of PVTT. So, the Chinese Association of Liver Cancer revised the 2016 version of consensus to adapt to the development of PVTT treatment.

Based on internationally accepted practice, the grades of evidence we use are presented in Table 1 [9]. We also adopted the United States Preventive Service Task Force recommendations to assign 5 alphabets (A-D, I) to denote the strength of recommendation for clinical practice (Table 2) [10]. Child-Pugh score was used for liver function evaluation in this edition, and ALBI score would be considered in the future as more and more evidence has become available.

\section{Consensus Recommendations}

\section{Diagnosis and Classification of PVTT}

PVTT is one of the most common complications of HCC. A diagnosis of HCC is a prerequisite to diagnose PVTT [11]. The imaging features of PVTT include solid lesions within the portal vein in all the phases of intravenous enhanced 3-phase computed tomography, especially with enhancement of contrast in the arterial phase and washout in the portal venous phase of the procedure $[12,13]$. Clinically, PVTT should be distinguished from portal vein thrombosis, which 
Cheng et al.: Chinese Expert Consensus on PVTT

Table 1. Grades of evidences

\begin{tabular}{|c|c|}
\hline $\begin{array}{l}\text { Grades of } \\
\text { evidences }\end{array}$ & Description \\
\hline Ia & Evidences are originated from the meta-analysis results of various RCTs \\
\hline $\mathrm{Ib}$ & Evidences are originated from the results of at least one well-designed RCT \\
\hline IIa & $\begin{array}{l}\text { Evidences are originated from the results of at least one well-designed perspective } \\
\text { non-RCT }\end{array}$ \\
\hline $\mathrm{IIb}$ & $\begin{array}{l}\text { Evidences are originated from the results of at least one well-designed interventional } \\
\text { clinical research of other type }\end{array}$ \\
\hline III & $\begin{array}{l}\text { Evidences are originated from the well-designed noninterventional clinical researches, } \\
\text { such as descriptive researches and relevant researches }\end{array}$ \\
\hline IV & $\begin{array}{l}\text { Evidences are originated from the reports made by committee of experts or the clinical } \\
\text { reports of authoritative experts }\end{array}$ \\
\hline
\end{tabular}

RCT, randomized controlled trial.

Table 2. Ranking of recommended opinion

\begin{tabular}{ll}
\hline $\begin{array}{l}\text { Grades of } \\
\text { evidences }\end{array}$ & Description \\
\hline A & $\begin{array}{l}\text { Favorable scientific evidences indicate that the medical treatment can provide clear and } \\
\text { definite benefits to the patients; physicians are strongly recommended to administer the } \\
\text { medical treatment to eligible patients }\end{array}$ \\
\hline B & $\begin{array}{l}\text { Existing evidences indicate that the medical treatment may provide moderate benefits } \\
\text { that outweigh the potential risks; physicians may suggest or patients may carry out the } \\
\text { said medical treatment }\end{array}$ \\
\hline C & $\begin{array}{l}\text { Existing evidences indicate that the medical treatment may provide only little benefits, or } \\
\text { the benefits do not outweigh the risks; physicians may suggest or administer the said } \\
\text { medical treatment selectively based on the patient's condition }\end{array}$ \\
\hline D & $\begin{array}{l}\text { Existing evidences indicate that the medical treatment would not benefit the patients, or } \\
\text { the potential risks would outweigh the benefits; physicians are recommended not to } \\
\text { administer the said medical treatment in patients }\end{array}$ \\
\hline I & $\begin{array}{l}\text { There are not enough scientific evidences, or the existing evidences cannot be used, to } \\
\text { evaluate the benefits and risks of the said medical treatment; physicians should help the } \\
\text { patients understand well the uncertainty of this medical treatment }\end{array}$ \\
&
\end{tabular}

occurs as a complication of cirrhosis or after splenectomy. Portal vein thrombosis is not enhanced in the arterial phase. It occasionally disappears or improves after anticoagulant therapy [14].

The extent of PVTT is closely related to prognosis of HCC. The HCC staging systems that are commonly used today are the TNM staging, BCLC staging, and Japanese integrated staging systems. All these staging systems accept the importance of PVTT. However, they do not further define the extent of PVTT. At present, there are 2 classifications for PVTT: the Japanese $V_{P}$ classification [15] and the Cheng's classification as suggested by Professor Cheng Shuqun of China [16-18].

The Cheng's classification comprises 4 levels based on the extent of tumor thrombus in the portal vein shown on medical imaging: type I, tumor thrombus involving segmental or sectoral branches of the portal vein or above; type II, tumor thrombus involving the right/left 


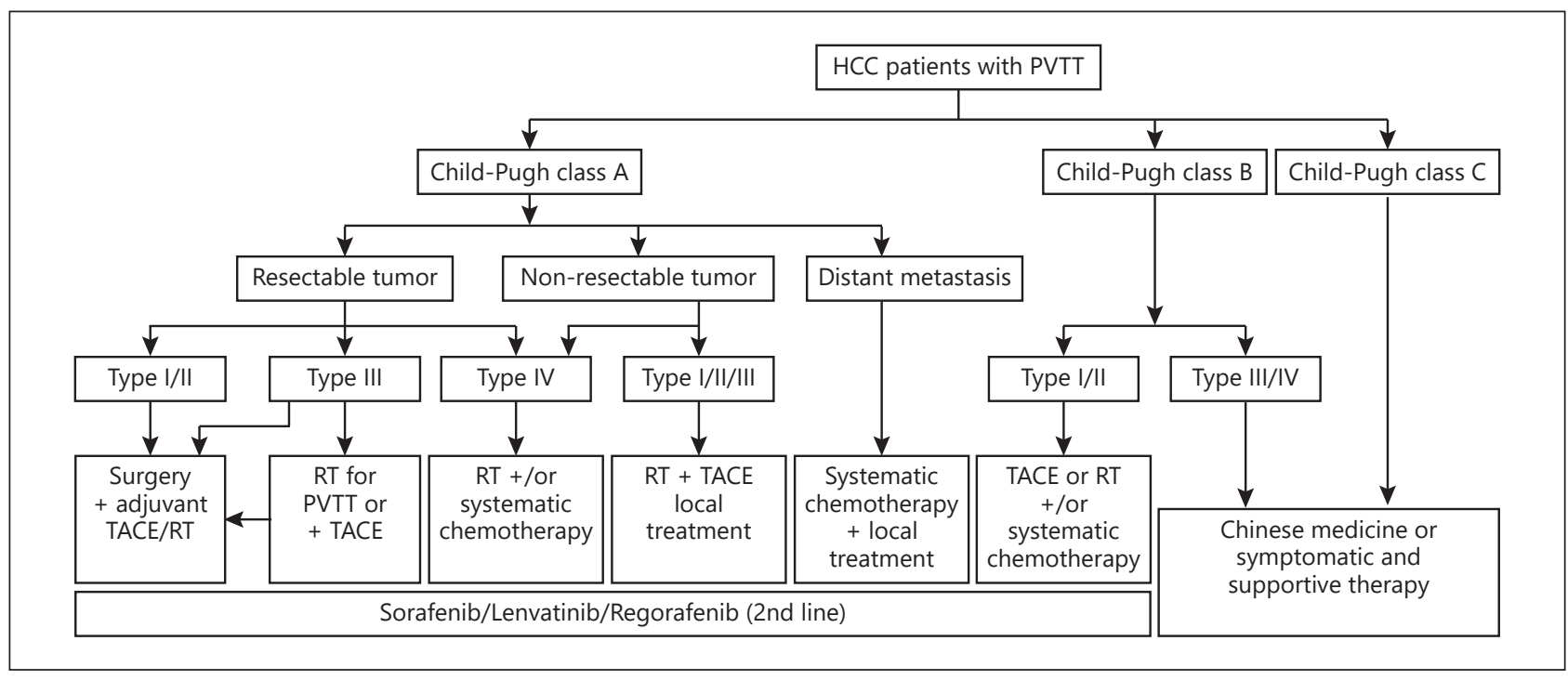

Fig. 1. Diagnosis and treatment of HCC with PVTT. HCC, hepatocellular carcinoma; PVTT, portal vein tumor thrombus; RT, radiotherapy; TACE, transcatheter arterial chemoembolization.

portal vein; type III, tumor thrombus involving the main portal vein; and type IV, tumor thrombus involving the superior mesenteric vein. Type $\mathrm{I}_{0}$, tumor thrombus found only under microscopy. Many studies have supported that the Cheng's classification to be more applicable than the $V_{P}$ classification for disease assessment, treatment selection, and prognostic judgment in patients with PVTT [17-19], and hence it is recommended to be used for classifying the extent of PVTT.

\section{Multidisciplinary Therapy Path for HCC with PVTT}

A multidisciplinary team to coordinate diagnosis and treatment of HCC patients with PVTT provides maximal benefits to patients. The therapeutic plan for the treatment of HCC with PVTT formulated by the National Research Cooperative Group for Diagnosis and Treatment of HCC with Tumor Thrombus is presented in Figure 1. Patients with Child-Pugh A liver function can undergo any treatment according to the PVTT type. When the lesion is resectable and when there is no extrahepatic metastasis, patients with type I/II PVTT should undergo surgical resection of the PVTT en bloc with the primary HCC. For patients with PVTT type III, the treatment choices include surgery, RT, and/or TACE depending on the patient's preference. For unresectable lesions, patients with type I/II/III PVTT should receive RT combined with TACE as the primary treatment, and patients with type IV PVTT should receive RT or systemic therapy. Patients with Child-Pugh B liver function should first receive antiviral treatment for HCC secondary to hepatitis B or C infections. If the liver function improves to Child-Pugh A, then these patient subgroups can be treated as mentioned above. Surgery and TACE are not recommended for Child-Pugh B patients. Child-Pugh C patients should only receive supportive care. Child-Pugh A and Child-Pugh B patients who have extrahepatic metastases can receive systemic chemotherapy and/or local treatment. Sorafenib and Lenvatinib can be used for patients with all extents of PVTT with Child-Pugh A liver function. Regorafenib is the second-line treatment of sorafenibresistant PVTT patients. 
Cheng et al.: Chinese Expert Consensus on PVTT

\section{Recommended First-Line Treatment Options for PVTT}

The treatment of HCC patients with PVTT is based on the patients' liver function, the stage of hepatic lesion, and the extent of PVTT. A strategy that can either eliminate or control HCC with PVTT using multimodality therapy can extend survival and improve quality of life of the patient.

\section{Surgery}

\section{Recommendations}

Surgery is the preferred treatment in patients with Child-Pugh A, PVTT type I/II, and ECOG PS 0-1 (Evidence level IIb, Recommendation A); type III PVTT patients can undergo surgery directly or after tumor downstaging using RT and/or TACE (Evidence level IIb, Recommendation B).

Adjuvant TACE after surgery can be used to reduce recurrence (Evidence level Ib, Recommendation B).

Surgical treatment is considered to be potentially curative and is the preferred treatment option for HCC patients with type I/II PVTT. En bloc resection of the primary HCC and PVTT provides a potential for cure. Many studies reported that patients who had undergone surgery had better prognosis than those treated with TACE $[11,20,21]$ or TACE combined with RT [22].

Type I/II PVTT are more suitable for resection than type III/IV (Evidence level IIb) [17, $23,24]$. En bloc resection can be performed in type I/II PVTT patients with partial hepatectomy or hemi-hepatectomy. For type III PVTT patients, as the PVTT has extended to the main portal vein, partial hepatectomy has to be combined with thrombectomy or main portal vein resection followed by reconstruction. At present, studies have revealed that there is no significant difference in prognosis among these surgical procedures (Evidence level IIb) [25]. Thrombectomy is by far the most commonly used surgical procedure.

The following are the recommendations for reducing recurrence rates and metastasis after surgery: (1) preoperative small-dose RT has been reported to downstage some type III PVTT patients, reduce recurrence rate without increasing surgical risks, and reduce postoperative hepatic failure rates (Evidence level IIa) [26]. (2) Adjuvant TACE after surgery has been reported to reduce recurrence rates and prolong survival of PVTT patients in a randomized controlled trial (from January 1996 to December 2004, including 126 patients) (Evidence level Ib) [27]; but a recent meta-analysis revealed that adjuvant TACE can only increase the 1-year survival rate. (3) Adjuvant hepatic artery infusion chemotherapy (HAIC) would be effective for recurrence prevention (IIb) [28].

Other treatment recommendations that are controversial include the following: (1) preoperative TACE has been reported to improve postoperative survival, but it may increase operative risks (Evidence level IIb) [29]. (2) There is a lack of high-level evidence for targeted therapy, adjuvant RT, or intravenous chemotherapy.

\section{Nonsurgical Therapies}

HAIC or TACE

Recommendations

- Patients with nonresectable primary tumor, type I/II PVTT, and Child-Pugh A liver function may receive HAIC (Evidence level Ib, Recommendation B), TACE (Evidence level IIb, Recommendation B), alone or in combination with RT (Evidence level IIb, Recommendation A). 


\section{Liver

Cheng et al.: Chinese Expert Consensus on PVTT

- $\quad$ Patients with Child-Pugh B liver function or type III/IV PVTT are not recommended to receive TACE (Evidence level IIb, Recommendation $\mathrm{C}$ ).

TACE is one of the most commonly used techniques to manage nonresectable HCC with PVTT [30]. Despite the possible benefit of TACE in prolonging overall survival (4-7 months) in patients with HCC and PVTT type III/IV, the use of TACE in patients is controversial due to the risk of liver infarction and hepatic failure [31]. At present, TACE is considered for PVTT patients with good liver function with adequate collateral circulation around the obstructed portal vein $[32,33]$. The overall survival rate varies greatly among patients with PVTT after TACE. The patient survival rates decreased from $82 \%$ at 3 months to $71 \%$ at 6 months and $47 \%$ at 12 months, with a median survival of 10 months. Patients with Child-Pugh A liver function had better median survival when compared to patients with Child-Pugh B (15 vs. 13 months) [34], and the complete remission rate, partial remission rate, and stable disease rate were reported to be $0,19.5-26.3$, and $42.5-62.7 \%$, respectively [35-37]. Lipiodol and gelatin sponge are common embolizing agents used in TACE [38]. Some reports have suggested that TACE, when combined with lipiodol, is more effective than TAI or conservative treatment $[30,39]$. The effectiveness of the embolizing agents depends on their size. The smaller the diameter of an embolizing agent, the better is the effect on PVTT patients and the lower is its adverse side effects [40,41]. The use of super-selective catheterization improves therapeutic effects and reduces damages to the normal liver when compared with conventional TACE. Recently, TACE with drug-eluting beads has been introduced into clinical application; however, its effects on HCC patients with PVTT are controversial [42].

HAIC was developed to treat metastatic liver tumors and was known to be more effective than conventional systemic chemotherapy. Recently, HAIC was then applied to advanced HCC [43]. A prospective randomized controlled study including 58 HCC patients with PVTT in Korea revealed that the median OS of HAIC group was 14.9 months, which was significantly higher than that of Sorafenib group (7.2 months, $p=0.012)$ [44].

\section{Radiotherapy}

\section{External Beam Radiation Therapy}

Recommendations

- Patients with nonresectable HCC with all types of PVTT, with Child-Pugh A or B liver function, are recommended to receive RT with the target region containing both the primary tumors and PVTT - 3-dimensional conformal RT or intensify-modulated RT (IMRT) 95\% plan target volume 40-60 Gy/2-3 Gy (Evidence level IIb, Recommendation B) or SBRT 36-40 Gy/5-6 Gy (Evidence level IIb, Recommendation A).

- Patients with Child-Pugh A liver function and types I, II, and III PVTT are recommended to receive combined RT and TACE (Evidence level Ib, Recommendation A). The RT target region includes the primary tumor and PVTT or only the PVTT.

With development of newer technologies such as 3-dimensional conformal RT, IMRT, and 3-dimensional oriented RT (SBRT), radiation dosage to the targeted regions can be increased while giving better protection to the adjacent healthy tissues [45-47]. This allows the maximum use of RT technologies and enables their use in HCC patients with all types of PVTT.

The use of RT alone or in combination with other treatment such as TACE improved survival and quality of life in HCC patients with PVTT. Yoon et al. [48] conducted a prospective randomized controlled study including $90 \mathrm{HCC}$ patients with PVTT and there were 45 cases in the TACE combined with RT group and 45 cases in the Sorafenib group. The results revealed 
Cheng et al.: Chinese Expert Consensus on PVTT

that the median OS of TACE combined with RT group was 12.8 months, which was significantly higher than that of Sorafenib group (10.0 months, $p=0.04)$.

Target localization suggests the use of computed tomography and MRI image fusion technology based on the area of lipiodol deposition after TACE. The clinical target volume is $4 \mathrm{~mm}$ larger than the diameter of the tumor area [49]. The plan target volume should be determined on the basis of a moving target, set-up error, and random error. The designation of the irradiation area is still controversial, which should be determined individually. The hepatic lesion and PVTT should be irradiated simultaneously if the hepatic lesion is small and PVTT is nearby. If the volume of the primary tumor is large or PVTT is distant to the primary tumor, only the PVTT should receive irradiation [50].

There is not enough evidence to determine the best radiation and fraction doses. The existing evidence suggests a positive correlation between total radiation dose and tumor response [51]. However, multivariate analysis only showed response to RT to be associated with survival [51,52]. Image-guided IMRT should be applied if available, which is better than conventional 3D-CRT [53].

Radiation-induced liver disease (RILD) or radiation hepatitis is a subacute form of liver injury, which occurs due to over exposure of the liver to radiation [54]. The key to prevent RILD is to keep the total dose within the tolerance range limit when designing the RT plan [54]. As most HCC patients in China have a cirrhotic background, the radiation tolerance dose of the liver in these patients is lower than that in patients from other countries. The liver tolerance dose (average dose of the liver) is 23 Gy for Child-Pugh A patients and only 6 Gy for Child-Pugh B patients [55]. The most common risk factors of RILD include preexisting poor liver function, high irradiation volume, coexisting PVT, and acute liver toxicity due to other causes $[54,55]$. It is reported that individualized adaptive RT based on a direct biomarker of liver function such as ICG 15 can be used to achieve both high rates of local control and a high degree of safety without sacrificing either (Evidence level IIa) [56].

Evidence from clinical studies has shown a combination of RT and TACE produces better clinical outcomes than TACE or RT alone. The time interval between TACE and RT should not exceed 1 month [57]. When TACE is combined with RT, the order of the treatments given should be decided clinically. As the effect on liver function is less in patients receiving RT first than those receiving TACE first, with similar treatment outcomes, RT should be given before TACE [58].

\section{Internal Radiation Therapy}

Recommendations

- Patients with nonresectable primary tumors; type I, II, and III PVTT; and Child-Pugh A liver function could be treated with transarterial arterial radio-embolization (TARE; Evidence level IIb, Recommendation C) or portal veins $\mathrm{I}^{125}$ seed implantation (Evidence level IIb, Recommendation B).

Patients treated with $\mathrm{I}^{125}$ particle seeds implanted in the portal vein and TACE have been reported to have better survival outcomes when compared to patients treated with TACE alone. This combination therapy also improved the reperfusion rate of portal vein significantly [59]. Another study showed ${ }^{125}$ seeds followed by TACE significantly improved the median survival and progression-free survival rates when compared to $\mathrm{I}^{125}$ alone $(p=0.037$ and 0.002 , respectively) [60]. TARE with yttrium-90 $\left(\mathrm{Y}^{90}\right)$ microspheres is considered to be a viable treatment option in HCC patients with PVTT. TARE has been shown to produce better long-term survival outcomes than TACE [61]. However, The SARAH trial revealed that the overall survival did not significantly differ between the Sorafenib group and TARE group for advanced HCC patients. [62]. Furthermore, there is no uniform dosage standard at present for internal radiation therapy. 
Cheng et al.: Chinese Expert Consensus on PVTT

\section{Systematic Therapy}

\section{Recommendations}

- Nucleoside analogs are recommended in patients with PVTT with positive HBV-DNA (Evidence level Ia, Recommendation A). Reactivation of HBV is of high importance in patients detected with negative HBV-DNA.

- Sorafenib and Lenvatinib are recommended as the basic drug for PVTT patients with Child-Pugh A liver function (Evidence level Ib, Recommendation A). Regorafenib is recommended as the second-line treatment for PVTT patients with Child-Pugh A liver function (Evidence level Ib, Recommendation A).

- Chemotherapy is recommended in PVTT patients (Evidence level IIb, Recommendation B) with extrahepatic metastasis and Child-Pugh A liver function.

Persistent HBV infection is an important poor risk factor for occurrence, progression, recurrence, and death in patients with HCC secondary to HBV infection. Antiviral therapy reduces postoperative recurrence and improves survival of HCC patients [63]. Antiviral therapy should also be given to PVTT patients $[64,65]$.

Sorafenib and Lenvatinib are universally accepted therapy that effectively prolongs survival in patients with advanced HCC (Evidence level Ib) [66, 67]. Both have been listed by the China Food and Drug Administration as the first-line treatment option in patients with advanced HCC. The STORM was a phase 3, double-blind, randomized, placebo-controlled study, which evaluated the effectiveness of sorafenib as adjuvant therapy to surgery. When compared to placebo, sorafenib did not show any significant improvement in the median recurrence-free survival (33.3 vs. 33.7 months, $p=0.26$ ), suggesting that adjuvant sorafenib to be ineffective [68]. The effectiveness of Sorafenib and TACE combination has also been controversial [69-71]. Regorafenib is recommended as the second-line treatment for PVTT patients (Evidence level Ib) [72].

The EACH study demonstrated that FOLFOX 4 (an oxaliplatin-containing chemotherapy) provided partial cure in patients with advanced HCC (including PVTT patients). FOLFOX 4 might be administered in patients with good liver function and tolerance (Evidence level Ib) [73]. A phased II prospective study revealed that mFOLFOX4 combined with Sorafenib would be more effective, but the results need further validation [74].

\section{Local Treatment}

\section{Recommendations}

- Local ablation therapies should be recommended in PVTT patients with caution; further studies are warranted (Evidence level III, Recommendation C). Local ablation therapies may be combined with TACE (Evidence level IIb, Recommendation B).

The local ablation therapies include percutaneous ethanol injection, radiofrequency ablation, and laser ablation. These therapies may be adopted to reduce tumor load and recanalization of portal vein. However, local therapies must be used cautiously as there is a risk of damaging the portal vein wall and bile duct. In addition, a high recurrence rate of PVTT has been reported within a short period of time (Level III evidence) $[75,76]$. 
Cheng et al.: Chinese Expert Consensus on PVTT

\section{Symptomatic and Supportive Treatment}

\section{Recommendations}

- Symptomatic and supportive treatment is recommended in patients with Child-Pugh C liver function, with massive ascites or gastrointestinal bleeding due to esophageal varices and hepatic encephalopathy (Evidence level Ia, Recommendation A).

Portal vein stenting may be adopted to recanalize blood flow in the portal veins of PVTT patients, with resultant increase in blood flow to the liver, but without reducing the tumor load. In patients with PVTT, portal vein stenting can result in improved liver functions, reduced portal vein pressure, and at the same time, win time for other therapies such as RT and TACE to act (Evidence level III) [77].

Most complications of PVTT result from portal hypertension. The common complications include upper gastrointestinal hemorrhage, ascites, hypersplenism, hepatorenal syndrome, and hepatic failure. For therapeutic methods, please refer to the article on treatment of portal hypertension [78]. In addition, Chinese medicine such as Huaier granule [79] could also be used for PVTT patients with nonresectable primary tumors.

\section{Future Outlook}

It is necessary to develop a treatment consensus in China as HCC patients with PVTT in China are different from those in Europe and America in terms of etiology and biological behavior. Although treatment of HCC patients with PVTT is still controversial, new evidences are being gathered. Similar to the multidisciplinary approach of HCC treatment in the United States (the American Association for the Study of Liver Diseases practice guidelines) and Europe (the European Association for the Study of the Liver - European Organization for Research and Treatment of Cancer) for HCC management, we have adopted a multidisciplinary approach for HCC with PVTT. This treatment approach when combined with early diagnosis will enable a larger number of patients to receive an appropriate treatment based on the stage of the disease.

In our consensus meetings, the following principles in clinical practice are emphasized: (1) Multidisciplinary treatment should be used in HCC patients with PVTT to achieve better results. (2) Prolongation of overall survival is the most important target, and the chance of cure is low. Emphasis should also be given to the quality of life of these patients. The treatment complication rate should be kept at a minimum. (3) Local treatment should be combined with systemic treatment to provide better long-term survival for these patients.

More RCTs should be conducted in HCC patients with PVTT. The molecular mechanisms underlying the genesis and development of PVTT also need to be studied to lay the foundation of more future effective treatment. The role of Chinese traditional medicine in the treatment of PVTT as an adjuvant to other therapeutic options such as surgical treatment, TACE, or RT should be evaluated.

\section{Acknowledgments}

The authors would like to acknowledge Prof. Lau Wan Yee for providing writing support and technical assistance in developing the English version of this consensus. Also we acknowledge the Consensus Revision Committee.

Committee members: Wei Ding, Xiao Hui Yu, Chi Dan Wan, Zhi Ming Wang, Jian Wang, Kui Wang, Lu Wang, Zhen Xia Wang, Yao Dong Wang, Yi Lei Mao, Tian Fu Wen, Chi Hua Fang, Tao Yin, Xue Tao Shi, Jie Shi, Shi Chun Lu, Qi Ping Lu, Sheng Long Ye, Li Guo Tian, Lin Hu Tong, Wen Ming Cong, Ming Kuang, Xin Yu Bi, Bin 
Cheng et al.: Chinese Expert Consensus on PVTT

Liu, Ji Ye Zhu, Yu Zhu, Tian QI Liu, Yong Xiong Liu, Lian Xin Liu, Yan Yan Liu, Jian Hua Liu, Ying Bin Liu, Zhao Hui Tang, Jun Hui Sun, Bei Cheng Sun, Hui Chuan Sun, Yi Ping Mou, Jiang Tao Li, Qi Yong Li, Jun Li, Bo Li, Xiang Cheng Li, Ai Jun Li, Jing Li, Bin Li, Ding Hua Yang, Zhen Yu Xiao, Li Qun Wu, Zheng Ping Yu, Min Gang Ying, Feng Shen, Tian Qiang Song, Zhi Wei Zhang, Xue Wen Zhang, Jian Jun Zhang, Shao Geng Zhang, Min Zhang, Ti Zhang, Qi Zhang, Cai De Lu, Wei Chen, Min Chen, Xin Shi Chen, Rui Fang Fan, Lin Lin, Dong Zhou, Cun Cai Zhou, Jie Zhou, Jian Zhou, Dong Hai Zheng, Ya Xin Zheng, Yan Meng, Ming Zhao, Wei Dong Jia, Yi Ren Hu, De Fei Hong, Lin Zhong, Ning Yang Jia, Yi Jun Xia, Jing Lin Xia, Li Xu, Xiao Yu Yin, Wei Xing Guo, Rong Ping Guo, Yu Fu Tang, Yang Qing Huang, Li Ping Cao, Wei Gong, Li Jian Liang, He Ping Peng, Bao Gang Peng, Shu You Peng, Xu Ting Zhi, Zhang Jun Cheng, Xian Bo Fu, Zhi Xiang Jian, Yang Cai, Jian Zhai, Le Qun Li, Gao Jun Teng, Zhi Ping Yan, Zuo Jun Zhen, Chao Liu Dai. Secretariats: Ju Xian Sun, Rong Ping Guo, Xin Yu Bi.

\section{Statement of Ethics}

Our manuscript complies with the guidelines for human studies and was conducted ethically in accordance with the World Medical Association Declaration of Helsinki. In the manuscript, all authors state that subjects have given their written informed consent and that the study protocol was approved by the institute's committee on human research.

\section{Disclosure Statement}

The authors have no conflicts of interest to declare.

\section{Funding Sources}

None.

\section{Author Contributions}

All the authors planned the study and contributed to the interpretation of the data, revisions, and gave input at all stages of the study. All the authors have approved the final version of the manuscript.

\section{References}

1 Torre LA, Bray F, Siegel RL, Ferlay J, Lortet-Tieulent J, Jemal A. Global cancer statistics, 2012. CA Cancer J Clin. 2015 Mar;65(2):87-108.

2 Chen W, Zheng R, Baade PD, Zhang S, Zeng H, Bray F, et al. Cancer statistics in China, 2015. CA Cancer J Clin. 2016 Mar-Apr;66(2):115-32.

3 Zhang ZM, Lai EC, Zhang C, Yu HW, Liu Z, Wan BJ, et al. The strategies for treating primary hepatocellular carcinoma with portal vein tumor thrombus. Int J Surg. 2015 Aug;20:8-16.

4 Pawarode A, Voravud N, Sriuranpong V, Kullavanijaya P, Patt YZ. Natural history of untreated primary hepatocellular carcinoma: a retrospective study of 157 patients. Am J Clin Oncol. 1998 Aug;21(4):386-91.

5 Li SH, Wei W, Guo RP, Shi M, Guo ZX, Chen ZY, et al. Long-term outcomes after curative resection for patients with macroscopically solitary hepatocellular carcinoma without macrovascular invasion and an analysis of prognostic factors. Med Oncol. 2013 Dec;30(4):696.

6 Li SH, Guo ZX, Xiao CZ, Wei W, Shi M, Chen ZY, et al. Risk factors for early and late intrahepatic recurrence in patients with single hepatocellular carcinoma without macrovascular invasion after curative resection. Asian Pac J Cancer Prev. 2013;14(8):4759-63.

7 Galle PR, Forner A, Llovet JM, Mazzaferro V, Piscaglia F, Raoul JL, et al.; European Association for the Study of the Liver. Electronic address: easloffice@easloffice.eu; European Association for the Study of the Liver. EASL Clinical Practice Guidelines: management of hepatocellular carcinoma. J Hepatol. 2018 Jul;69(1):182-236.

8 Cheng S, Chen M, Cai J; National Research Cooperative Group for Diagnosis and Treatment of Hepatocellular Carcinoma with Tumor Thrombus. Chinese expert consensus on multidisciplinary diagnosis and treatment of hepatocellular carcinoma with portal vein tumor thrombus: 2016 edition. Oncotarget. 2017 Jan;8(5):886776. 
9 Ryder SD; British Society of Gastroenterology. Guidelines for the diagnosis and treatment of hepatocellular carcinoma (HCC) in adults. Gut. 2003 May;52(Suppl 3):iii1-8.

10 U.S. Preventive Services Task Force. Grade Definitions and Suggestions for Practice. 2012; Available from: http://www.uspreventiveservicestaskforce.org/Page/Name/grade-definitions.

11 Wang K, Guo WX, Chen MS, Mao YL, Sun BC, Shi J, et al. Multimodality treatment for hepatocellular carcinoma with portal vein tumor thrombus: a large-scale, multicenter, propensity mathching score analysis. Medicine (Baltimore). 2016 Mar;95(11):e3015.

12 Qin S; Primary Liver Cancer Diagnosis and Treatment Expert Panel of the Chinese Ministry of Health. Guidelines on the diagnosis and treatment of primary liver cancer (2011 edition). Chin Clin Oncol. 2012 Sep;1(1):10.

13 Hennedige T, Venkatesh SK. Advances in computed tomography and magnetic resonance imaging of hepatocellular carcinoma. World J Gastroenterol. 2016 Jan;22(1):205-20.

14 Ponziani FR, Zocco MA, Campanale C, Rinninella E, Tortora A, Di Maurizio L, et al. Portal vein thrombosis: insight into physiopathology, diagnosis, and treatment. World J Gastroenterol. 2010 Jan;16(2):143-55.

15 Ikai I, Yamamoto Y, Yamamoto N, Terajima H, Hatano E, Shimahara Y, et al. Results of hepatic resection for hepatocellular carcinoma invading major portal and/or hepatic veins. Surg Oncol Clin N Am. 2003 Jan;12(1):65-75.

16 Shuqun C, Mengchao W, Han C, Feng S, Jiahe Y, Guanghui D, et al. Tumor thrombus types influence the prognosis of hepatocellular carcinoma with the tumor thrombi in the portal vein. Hepatogastroenterology. 2007 Mar;54(74):499-502.

17 Shi J, Lai EC, Li N, Guo WX, Xue J, Lau WY, et al. Surgical treatment of hepatocellular carcinoma with portal vein tumor thrombus. Ann Surg Oncol. 2010 Aug;17(8):2073-80.

18 Shi J, Lai EC, Li N, Guo WX, Xue J, Lau WY, et al. A new classification for hepatocellular carcinoma with portal vein tumor thrombus. J Hepatobiliary Pancreat Sci. 2011 Jan;18(1):74-80.

19 Niu ZJ, Ma YL, Kang P, Ou SQ, Meng ZB, Li ZK, et al. Transarterial chemoembolization compared with conservative treatment for advanced hepatocellular carcinoma with portal vein tumor thrombus: using a new classification. Med Oncol. 2012 Dec;29(4):2992-7.

20 Peng ZW, Guo RP, Zhang YJ, Lin XJ, Chen MS, Lau WY. Hepatic resection versus transcatheter arterial chemoembolization for the treatment of hepatocellular carcinoma with portal vein tumor thrombus. Cancer. 2012 Oct;118(19):4725-36.

21 Liang L, Chen TH, Li C, Xing H, Han J, Wang MD, et al. A systematic review comparing outcomes of surgical resection and non-surgical treatments for patients with hepatocellular carcinoma and portal vein tumor thrombus. HPB (Oxford). 2018 Dec;20(12):1119-29.

22 Yu JI, Choi GS, Lim DH, Lee E, Joh JW, Kwon CH, et al. Treatment of Naïve HCC Combined with Segmental or Subsegmental Portal Vein Tumor Thrombosis: Liver Resection Versus TACE Followed by Radiotherapy. Anticancer Res. 2018 Aug;38(8):4919-25.

23 Chen XP, Qiu FZ, Wu ZD, Zhang ZW, Huang ZY, Chen YF, et al. Effects of location and extension of portal vein tumor thrombus on long-term outcomes of surgical treatment for hepatocellular carcinoma. Ann Surg Oncol. 2006 Jul;13(7):940-6.

24 Cao JY, Wang ZS, Wu SK, Yu Y, Zhu C, Wu L. Analysis of surgical treatment and prognostic factors for hepatocellular carcinoma with portal vein tumor thrombus. Transl Cancer Res. 2017;6(1):247-53.

25 Chok KS, Cheung TT, Chan SC, Poon RT, Fan ST, Lo CM. Surgical outcomes in hepatocellular carcinoma patients with portal vein tumor thrombosis. World J Surg. 2014 Feb;38(2):490-6.

26 Li N, Feng S, Xue J, Wei XB, Shi J, Guo WX, et al. Hepatocellular carcinoma with main portal vein tumor thrombus: a comparative study comparing hepatectomy with or without neoadjuvant radiotherapy. HPB (Oxford). 2016 Jun;18(6):549-56.

27 Peng BG, He Q, Li JP, Zhou F. Adjuvant transcatheter arterial chemoembolization improves efficacy of hepatectomy for patients with hepatocellular carcinoma and portal vein tumor thrombus. Am J Surg. 2009 Sep; 198(3):313-8.

28 Hatano E, Uemoto S, Yamaue H, Yamamoto M; Japanese Society of Hepato-Biliary-Pancreatic Surgery. Significance of hepatic resection and adjuvant hepatic arterial infusion chemotherapy for hepatocellular carcinoma with portal vein tumor thrombus in the first branch of portal vein and the main portal trunk: a project study for hepatic surgery of the Japanese Society of Hepato-Biliary-Pancreatic Surgery. J Hepatobiliary Pancreat Sci. 2018 Sep;25(9):395-402.

29 Yoshidome H, Takeuchi D, Kimura F, Shimizu H, Ohtsuka M, Kato A, et al. Treatment strategy for hepatocellular carcinoma with major portal vein or inferior vena cava invasion: a single institution experience. J Am Coll Surg. 2011 May;212(5):796-803.

30 Xue TC, Xie XY, Zhang L, Yin X, Zhang BH, Ren ZG. Transarterial chemoembolization for hepatocellular carcinoma with portal vein tumor thrombus: a meta-analysis. BMC Gastroenterol. 2013 Apr;13(1):60.

31 Chan SL, Chong CC, Chan AW, Poon DM, Chok KS. Management of hepatocellular carcinoma with portal vein tumor thrombosis: review and update at 2016. World J Gastroenterol. 2016 Aug;22(32):7289-300.

32 Chung GE, Lee JH, Kim HY, Hwang SY, Kim JS, Chung JW, et al. Transarterial chemoembolization can be safely performed in patients with hepatocellular carcinoma invading the main portal vein and may improve the overall survival. Radiology. 2011 Feb;258(2):627-34.

33 Kim HC, Chung JW, Lee W, Jae HJ, Park JH. Recognizing extrahepatic collateral vessels that supply hepatocellular carcinoma to avoid complications of transcatheter arterial chemoembolization. Radiographics. 2005 0ct; 25 Suppl 1:S25-39. 
Cheng et al.: Chinese Expert Consensus on PVTT

34 Ajit Y, Sudarsan H, Saumya G, Abhishek A, Navneet R, Piyush R, et al. Transarterial chemoembolization in unresectable hepatocellular carcinoma with portal vein thrombosis: a perspective on survival. Oman Med J. 2014 Nov;29(6):430-6.

35 Liu L, Zhang C, Zhao Y, Qi X, Chen H, Bai W, et al. Transarterial chemoembolization for the treatment of advanced hepatocellular carcinoma with portal vein tumor thrombosis: prognostic factors in a single-center study of 188 patients. BioMed Res Int. 2014;2014:194278.

36 Jang JW, Bae SH, Choi JY, Oh HJ, Kim MS, Lee SY, et al. A combination therapy with transarterial chemo-lipiodolization and systemic chemo-infusion for large extensive hepatocellular carcinoma invading portal vein in comparison with conservative management. Cancer Chemother Pharmacol. 2007 Jan;59(1):9-15.

37 Luo J, Guo RP, Lai EC, Zhang YJ, Lau WY, Chen MS, et al. Transarterial chemoembolization for unresectable hepatocellular carcinoma with portal vein tumor thrombosis: a prospective comparative study. Ann Surg Oncol. 2011 Feb;18(2):413-20.

38 Liu YS, Ou MC, Tsai YS, Lin XZ, Wang CK, Tsai HM, et al. Transarterial chemoembolization using gelatin sponges or microspheres plus lipiodol-doxorubicin versus doxorubicin-loaded beads for the treatment of hepatocellular carcinoma. Korean J Radiol. 2015 Jan-Feb;16(1):125-32.

39 Liu YM, Qin H, Wang CB, Fang XH, Ma QY. [Comparision of different interventional therapies for primary liver cancer]. Zhonghua Zhong Liu Za Zhi. 2007 Mar;29(3):232-5.

40 Chern MC, Chuang VP, Liang CT, Lin ZH, Kuo TM. Transcatheter arterial chemoembolization for advanced hepatocellular carcinoma with portal vein invasion: safety, efficacy, and prognostic factors. J Vasc Interv Radiol. 2014 Jan;25(1):32-40.

41 Tsochatzis EA, Fatourou E, O’Beirne J, Meyer T, Burroughs AK. Transarterial chemoembolization and bland embolization for hepatocellular carcinoma. World J Gastroenterol. 2014 Mar;20(12):3069-77.

42 Brown KT, Do RK, Gonen M, Covey AM, Getrajdman GI, Sofocleous CT, et al. Randomized trial of hepatic artery embolization for hepatocellular carcinoma using doxorubicin-eluting microspheres compared with embolization with microspheres alone. J Clin Oncol. 2016 Jun;34(17):2046-53.

43 Lyu N, Kong Y, Mu L, Lin Y, Li J, Liu Y, et al. Hepatic arterial infusion of oxaliplatin plus fluorouracil/leucovorin vs. sorafenib for advanced hepatocellular carcinoma. J Hepatol. 2018 Jul;69(1):60-9.

44 Choi JH, Chung WJ, Bae SH, Song DS, Song MJ, Kim YS, et al. Randomized, prospective, comparative study on the effects and safety of sorafenib vs. hepatic arterial infusion chemotherapy in patients with advanced hepatocellular carcinoma with portal vein tumor thrombosis. Cancer Chemother Pharmacol. 2018 Sep;82(3):469-78.

45 Hsieh CH, Liu CY, Shueng PW, Chong NS, Chen CJ, Chen MJ, et al. Comparison of coplanar and noncoplanar intensity-modulated radiation therapy and helical tomotherapy for hepatocellular carcinoma. Radiat Oncol. 2010 May;5(1):40.

46 Tang QH, Li AJ, Yang GM, Lai EC, Zhou WP, Jiang ZH, et al. Surgical resection versus conformal radiotherapy combined with TACE for resectable hepatocellular carcinoma with portal vein tumor thrombus: a comparative study. World J Surg. 2013 Jun;37(6):1362-70.

47 Kang J, Nie Q, Du R, Zhang L, Zhang J, Li Q, et al. Stereotactic body radiotherapy combined with transarterial chemoembolization for hepatocellular carcinoma with portal vein tumor thrombosis. Mol Clin Oncol. 2014 Jan;2(1):43-50.

48 Yoon SM, Ryoo BY, Lee SJ, Kim JH, Shin JH, An JH, et al. Efficacy and Safety of Transarterial Chemoembolization Plus External Beam Radiotherapy vs Sorafenib in Hepatocellular Carcinoma With Macroscopic Vascular Invasion: A Randomized Clinical Trial. JAMA Oncol. 2018 May;4(5):661-9.

49 Wang MH, Ji Y, Zeng ZC, Tang ZY, Fan J, Zhou J, et al. Impact factors for microinvasion in patients with hepatocellular carcinoma: possible application to the definition of clinical tumor volume. Int J Radiat Oncol Biol Phys. 2010 Feb;76(2):467-76.

50 Yu JI, Park HC. Radiotherapy as valid modality for hepatocellular carcinoma with portal vein tumor thrombosis. World J Gastroenterol. 2016 Aug;22(30):6851-63.

51 Huang BS, Tsang NM, Lin SM, Lin DY, Lien JM, Lin CC, et al. High-dose hypofractionated X-ray radiotherapy for hepatocellular carcinoma: tumor responses and toxicities. Oncol Lett. 2013 Nov;6(5):1514-20.

52 Xi M, Zhang L, Zhao L, Li QQ Guo SP, Feng ZZ, et al. Effectiveness of stereotactic body radiotherapy for hepatocellular carcinoma with portal vein and/or inferior vena cava tumor thrombosis. PLoS One. 2013 May;8(5):e63864.

53 Hou JZ, Zeng ZC, Wang BL, Yang P, Zhang JY, Mo HF. High dose radiotherapy with image-guided hypo-IMRT for hepatocellular carcinoma with portal vein and/or inferior vena cava tumor thrombi is more feasible and efficacious than conventional 3D-CRT. Jpn J Clin Oncol. 2016 Apr;46(4):357-62.

54 Benson R, Madan R, Kilambi R, Chander S. Radiation induced liver disease: A clinical update. J Egypt Natl Canc Inst. 2016 Mar;28(1):7-11.

55 Liang SX, Zhu XD, Xu ZY, Zhu J, Zhao JD, Lu HJ, et al. Radiation-induced liver disease in three-dimensional conformal radiation therapy for primary liver carcinoma: the risk factors and hepatic radiation tolerance. Int J Radiat Oncol Biol Phys. 2006 Jun;65(2):426-34.

56 Feng M, Suresh K, Schipper MJ, Bazzi L, Ben-Josef E, Matuszak MM, et al. Individualized Adaptive Stereotactic Body Radiotherapy for Liver Tumors in Patients at High Risk for Liver Damage: A Phase 2 Clinical Trial. JAMA Oncol. 2018 Jan;4(1):40-7.

57 Li XL, Guo WX, Hong XD, Yang L, Wang K, Shi J, et al. Efficacy of the treatment of transarterial chemoembolization combined with radiotherapy for hepatocellular carcinoma with portal vein tumor thrombus: A propensity score analysis. Hepatol Res. 2016 Oct; 46(11):1088-98. 
58 Li X, Guo W, Guo L, Lau WY, Ge N, Wang K, et al. Should transarterial chemoembolization be given before or after intensity-modulated radiotherapy to treat patients with hepatocellular carcinoma with portal vein tumor thrombus? a propensity score matching study. Oncotarget. 2018 May;9(36):24537-47.

59 Yang M, Fang Z, Yan Z, Luo J, Liu L, Zhang W, et al. Transarterial chemoembolisation (TACE) combined with endovascular implantation of an iodine- 125 seed strand for the treatment of hepatocellular carcinoma with portal vein tumour thrombosis versus TACE alone: a two-arm, randomised clinical trial. J Cancer Res Clin Oncol. 2014 Feb;140(2):211-9.

60 Li WW, Dai ZY, Wan HG, Yao LZ, Zhu J, Li CL, et al. [Endovascular implantation of iodine-125 seeds strand and portal vein stenting followed by transcatheter arterial chemoembolization combined therapy with sorafenib for hepatocellular carcinoma with main portal vein tumor thrombus]. Zhonghua Yi Xue Za Zhi. 2016 Jun; 96(23):1838-42.

61 Lau WY, Sangro B, Chen PJ, Cheng SQ, Chow P, Lee RC, et al. Treatment for hepatocellular carcinoma with portal vein tumor thrombosis: the emerging role for radioembolization using yttrium-90. Oncology. 2013;84(5): 311-8.

62 Vilgrain V, Pereira H, Assenat E, Guiu B, Ilonca AD, Pageaux GP, et al.; SARAH Trial Group. Efficacy and safety of selective internal radiotherapy with yttrium-90 resin microspheres compared with sorafenib in locally advanced and inoperable hepatocellular carcinoma (SARAH): an open-label randomised controlled phase 3 trial. Lancet Oncol. 2017 Dec;18(12):1624-36.

63 Yin J, Li N, Han Y, Xue J, Deng Y, Shi J, et al. Effect of antiviral treatment with nucleotide/nucleoside analogs on postoperative prognosis of hepatitis B virus-related hepatocellular carcinoma: a two-stage longitudinal clinical study. J Clin Oncol. 2013 Oct;31(29):3647-55.

64 Tsuda Y, Kobayashi S, Tomimaru Y, Akita H, Hama N, Wada H, et al. [Long-term survival of a patient with hepatocellular carcinoma with portal vein tumor thrombus treated with interferon- $\alpha$ and 5-fluorouracil combination therapy]. Gan To Kagaku Ryoho. 2013 Nov;40(12):1804-6.

65 Huang G, Lai EC, Lau WY, Zhou WP, Shen F, Pan ZY, et al. Posthepatectomy HBV reactivation in hepatitis B-related hepatocellular carcinoma influences postoperative survival in patients with preoperative low HBV-DNA levels. Ann Surg. 2013 Mar;257(3):490-505.

66 Bruix J, Raoul JL, Sherman M, Mazzaferro V, Bolondi L, Craxi A, et al. Efficacy and safety of sorafenib in patients with advanced hepatocellular carcinoma: subanalyses of a phase III trial. J Hepatol. 2012 Oct;57(4):821-9.

67 Kudo M, Finn RS, Qin S, Han KH, Ikeda K, Piscaglia F, et al. Lenvatinib versus sorafenib in first-line treatment of patients with unresectable hepatocellular carcinoma: a randomised phase 3 non-inferiority trial. Lancet. 2018 Mar;391(10126):1163-73.

68 Bruix J, Takayama T, Mazzaferro V, Chau GY, Yang J, Kudo M, et al.; STORM investigators. Adjuvant sorafenib for hepatocellular carcinoma after resection or ablation (STORM): a phase 3, randomised, double-blind, placebo-controlled trial. Lancet Oncol. 2015 Oct;16(13):1344-54.

69 Kokudo T, Hasegawa K, Matsuyama Y, Takayama T, Izumi N, Kadoya M, et al.; Liver Cancer Study Group of Japan. Survival benefit of liver resection for hepatocellular carcinoma associated with portal vein invasion. J Hepatol. 2016 Nov;65(5):938-43.

70 Zhu K, Chen J, Lai L, Meng X, Zhou B, Huang W, et al. Hepatocellular carcinoma with portal vein tumor thrombus: treatment with transarterial chemoembolization combined with sorafenib-a retrospective controlled study. Radiology. 2014 Jul;272(1):284-93.

71 Lencioni R, Llovet JM, Han G, Tak WY, Yang J, Guglielmi A, et al. Sorafenib or placebo plus TACE with doxorubicin-eluting beads for intermediate stage HCC: the SPACE trial. J Hepatol. 2016 May; 64(5):1090-8.

72 Bruix J, Qin S, Merle P, Granito A, Huang YH, Bodoky G, et al.; RESORCE Investigators. Regorafenib for patients with hepatocellular carcinoma who progressed on sorafenib treatment (RESORCE): a randomised, doubleblind, placebo-controlled, phase 3 trial. Lancet. 2017 Jan;389(10064):56-66.

73 Qin S, Bai Y, Lim HY, Thongprasert S, Chao Y, Fan J, et al. Randomized, multicenter, open-label study of oxaliplatin plus fluorouracil/leucovorin versus doxorubicin as palliative chemotherapy in patients with advanced hepatocellular carcinoma from Asia. J Clin Oncol. 2013 Oct;31(28):3501-8.

74 Goyal L, Zheng H, Abrams TA, Miksad R, Bullock AJ, Allen JN, et al. A Phase II and Biomarker Study of Sorafenib Combined with Modified FOLFOX in Patients with Advanced Hepatocellular Carcinoma. Clin Cancer Res. 2019 Jan;25(1):80-9.

75 Zheng JS, Long J, Sun B, Lu NN, Fang D, Zhao LY, et al. Transcatheter arterial chemoembolization combined with radiofrequency ablation can improve survival of patients with hepatocellular carcinoma with portal vein tumour thrombosis: extending the indication for ablation? Clin Radiol. 2014 Jun;69(6):e253-63.

76 Lu ZH, Shen F, Yan ZL, Li J, Yang JH, Zong M, et al. Treatment of portal vein tumor thrombus of hepatocellular carcinoma with percutaneous laser ablation. J Cancer Res Clin Oncol. 2009 Jun;135(6):783-9.

77 Vibert E, Azoulay D, Cunha AS, Adam R, Samuel D, Castaing D. Portal stenting for hepatocellular carcinoma extending into the portal vein in cirrhotic patients. J Surg Oncol. 2013 Jun;107(7):696-701.

78 de Franchis R, Baveno VI; Baveno VI Faculty. Expanding consensus in portal hypertension: Report of the Baveno VI Consensus Workshop: Stratifying risk and individualizing care for portal hypertension. J Hepatol. 2015 Sep;63(3):743-52.

79 Chen Q, Shu C, Laurence AD, Chen Y, Peng BG, Zhen ZJ, et al. Effect of Huaier granule on recurrence after curative resection of HCC: a multicentre, randomised clinical trial. Gut. 2018 Nov;67(11):2006-16. 